\title{
Frequency drifts of 3-min oscillations in microwave and EUV emission above sunspots
}

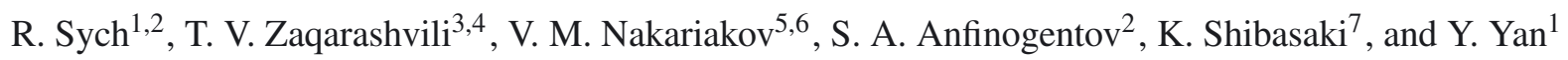 \\ ${ }^{1}$ Key Laboratory of Solar Activity, National Astronomical Observatories, Chinese Academy of Sciences, Beijing 100012, PR China \\ e-mail: sych@iszf.irk.ru \\ 2 Institute of Solar-Terrestrial Physics, Russian Academy of Sciences, Siberian Branch, 126a Lermontov st., Irkutsk 664033, Russia \\ 3 Space Research Institute, Austrian Academy of Sciences, Schmiedlstrasse 6, 8042 Graz, Austria \\ 4 Abastumani Astrophysical Observatory at Ilia State University, Kazbegi ave. 2a, Tbilisi, Georgia \\ 5 Centre for Fusion, Space and Astrophysics, Department of Physics, University of Warwick, Coventry CV4 7AL, UK \\ ${ }^{6}$ Central Astronomical Observatory at Pulkovo of the Russian Academy of Sciences, 196140 St Petersburg, Russia \\ 7 Nobeyama Solar Radio Observatory/NAOJ, Nagano 384-1305, Japan
}

Received 14 October 2011 / Accepted 5 November 2011

\begin{abstract}
Aims. We analysed 3-min oscillations of microwave and extreme ultraviolet (EUV) emission generated at different heights of a sunspot atmosphere, studied the amplitude and frequency modulation of the oscillations, and its relationship with the variation of the spatial structure of the oscillations.

Methods. High-resolution data obtained with the Nobeyama Radioheliograph, TRACE and SDO/AIA were analysed with pixelised wavelet filtering (PWF) and wavelet skeleton techniques.

Results. Three-minute oscillations in sunspots appear in the form of recurring trains of 8-20 min duration (13 min in average). The typical interval between the trains is 30-50 min. The oscillation trains are transient in frequency and power. The relative amplitude of 3-min oscillations was about 3-8\% and sometimes reached 17\%. Recurring frequency drifts of 3-min oscillations were detected during the development of individual trains, with the period varying in the range 90-240 s. A wavelet analysis showed that there are three types of oscillation trains: with positive drifts (to high frequencies), negative drifts, and without a drift. Negative drifts, i.e., when the 3-min oscillation period gradually increases, were found to occur more often. The start and end of the drifts coincides with the start time and end of the train. Sometimes two drifts co-exist, i.e. during the end of the previous drift, a new drift appears near $160 \mathrm{~s}$, when the frequency is in the low-frequency part of the 3-min spectrum, near $200 \mathrm{~s}$. This behaviour is seen at all levels of the sunspot atmosphere. The speed of the drift is $4-5 \mathrm{mHz} / \mathrm{h}$ in the photosphere, $5-8 \mathrm{mHz} / \mathrm{h}$ in the chromosphere, and $11-13 \mathrm{mHz} / \mathrm{h}$ in the corona. There were also low-frequency peaks in the spectrum, corresponding to the periods of 10-20 min, and 30-60 min. The comparative study of the spatial structure of 3-min oscillations in microwave and EUV shows the appearance of new sources of the sunspot oscillations during the development of the trains.

Conclusions. These structures can be interpreted as waveguides that channel upward propagating waves, which in turn are responsible for the 3-min oscillations. A possible explanation of the observed properties are two simultaneously operating factors: dispersive evolution of the upward propagating wave pulses and the non-uniformity of the oscillation power distribution over the sunspot umbra with different wave sources that correspond to different magnetic flux tubes with different physical conditions and line-of-sight angles.
\end{abstract}

Key words. Sun: oscillations - Sun: radio radiation - Sun: UV radiation - sunspots

\section{Introduction}

The analysis of multi-wavelength data obtained with the new generation of solar observational tools allows us to study the dynamics of the solar atmosphere at different heights with high spatial, spectral and temporal resolution. One of the interesting topics are 3-min oscillations in sunspots (e.g. Orrall 1966; Elliott 1969; Liu 1974). Fluctuations in the 3-min range are relatively weak at the photospheric level of sunspots, but they are significantly enhanced in their chromospheres (Lites 1986; Bard \& Carlsson 1997; Tziotziou et al. 2002; Kobanov et al. 2008, 2011) and in the transition region (Gurman et al. 1982; Lites 1992; Bogdan 2000). Observations of the photospheric magnetic field oscillations (Norton et al. 1999; Balthasar et al. 1998; Bellot Rubio et al. 2000) show a fine fragmentation of the magnetic field in the regions where the main power of fluctuations is localised inside sunspots. For example, optical observations show that 5-min oscillations of the magnetic field are mainly concentrated in the isolated tubes of magnetic field (pores) outside the big sunspots, as well as at the umbra-penumbra boundary (Zhugzhda et al. 2000). Blinkers or explosive events are also observed above sunspots with a similar time scale, from 400 to 1600 s (Harrison et al. 1999; Brković et al. 2000).

The first observations of the fine spatial structure of oscillating radio sources above strong sunspots at the transition region level were obtained by Gelfreikh et al. (1999) with the Nobeyama Radioheliograph. That study showed that the main source of broadband oscillations is concentrated in the centre of the source of the polarised radio emission at $17 \mathrm{GHz}$, which is coincident with the sunspots umbra. Subsequent investigations in the radio band (Shibasaki et al. 2001) using the correlation curves and images of radio sources showed that 3-min 
oscillations are present in sunspots for long periods. It was concluded that the brightness fluctuations of the radio sources could be caused by the density and temperature perturbations in acoustic waves that propagate through the third gyroresonant level (corresponding to the magnetic field of about $2 \mathrm{kG}$ ).

Nindos et al. (2002) used the difference radio maps obtained by VLA at 8.5 and $5 \mathrm{GHz}$ with high spatial resolution. They showed that the amplitude of 3-min oscillations varies irregularly in time, with a rapid rise phase, and a fairly slow decay phase. The spatial sources of the amplitude variations are localised in fine patches of a circular shape.

Sych \& Nakariakov (2008) studied the spatial, temporal and phase behaviour of sunspot oscillations, applying the method of pixelised wavelet filtering (PWF). The authors showed that the source of 3-min oscillations is located in the centre of the microwave source, which coincides with the sunspot umbra. On the other hand, 5-min oscillations were found to be mainly located in small, symmetric patches at the the umbra-penumbra boundary, similar to the findings obtained with VLA. Oscillations in different patches were found to have different phases.

The phenomenological relationship between 3-min oscillations of the radio flux from a sunspot and quasi-periodic bursts of energy release in coronal active regions nearby was established by Sych et al. (2009) and Nakariakov et al. (2010). Recently, Sych et al. (2010) used the system for automatic detection of solar oscillations (http://pwf.iszf.irk.ru), which showed that the power of 5-min oscillations in sunspots is concentrated in ring-shaped sources at the upper chromosphere or transition region, which coincide with the footpoints of coronal extreme ultraviolet (EUV) fans. These spatial details are associated with both the standing waves (symmetric spatial patches) and propagating waves (seen as transient, about 10-30 min, $V$-shaped increases in the spatial distribution of the oscillation power). The amplitude increase was seen simultaneously in microwaves and in EUV.

Rendtel et al. (2003) detected variable changes (drifts) of the periods of sunspot oscillations, using the SOHO/SUMER spectrograph (CIV, NeVIII) and EIT, $171 \AA$. The fluctuations of the Doppler shift and the emission intensity occur in the same frequency range, 3-7 $\mathrm{mHz}$, with a significant time-drift to higher frequencies. Most of the fluctuations in the transition region and lower corona are concentrated near the frequency of $6.2 \mathrm{mHz}$ and form oscillation trains that laste from 10 to $20 \mathrm{~min}$, sometimes up to $40 \mathrm{~min}$. A possible explanation of the drifts is the assumption that the curvature of the magnetic tubes above the sunspot leads to different directions of magnetohydrodynamic (MHD) wave propagation relative to the observer and, accordingly, to the periodic changes of the Doppler velocity and the sign of the frequency drift. Fludra (2001) found a significant frequency drift of $5.8 \mathrm{mHz}$ oscillations above sunspots in the transition region lines with $\mathrm{SOHO} / \mathrm{CDS}$. The typical duration of the train was found to be about $15 \mathrm{~min}$. Different frequencies in the spectrum were found to have different spatially separated sources.

Two main mechanisms were suggested to explain the 3-min sunspot oscillations: a chromospheric cavity (or resonator) and oscillations at the acoustic cut-off frequency. The first mechanism implies that the chromosphere is a resonant cavity for waves, which are reflected from the photosphere because of the sharp density gradient and from the transition region due to the sharp temperature gradient (Leibacher \& Stein 1981; Leibacher et al. 1982). It was recently shown numerically that quasi-monochromatic acoustic oscillations in the 3-min band are a natural response of the chromospheric resonator to a broadband (e.g., impulsive) excitation (Botha et al. 2011). The second mechanism implies that the 3-min oscillations result from the wake behind propagating disturbances in the stratified atmosphere, which oscillates at the acoustic cut-off frequency (Fleck \& Schmitz 1991; Kalkofen et al. 1994; Sutmann $\&$ Ulmschneider 1995; Kuridze et al. 2009). The nonlinear wake behind propagating acoustic pulses may develop in quasiperiodic shocks, which may shape the plasma properties in the chromosphere and the lower corona. These quasi-periodic shocks have been recently suggested as a mechanism for the quasi-periodic appearance of solar spicules and 5-min oscillations in intensity observed in the solar corona (Murawski \& Zaqarashvili 2010; Zaqarashvili et al. 2011).

In this paper we present results of a comprehensive study of 3-min oscillations at different heights over sunspots with the use of high-resolution EUV and microwave data obtained with SDO/AIA, TRACE and NoRH. The aim of the paper is to reveal the fine spatial-temporal structure of 3-min oscillations, contributing to the understanding of physical mechanisms for their generation and developing plasma diagnostics techniques. In Sect. 2 we describe the instruments and the analysed datasets. Section 3 describes the methods used in the analysis. Section 4 describes the results obtained. Sections 5 and 6 present discussion and conclusions, respectively.

\section{Instruments and observations}

To study oscillations in the microwave emission from sunspots, we used the Nobeyama Radioheliograph (NoRH, see Nakajima 1994) data at $17 \mathrm{GHz}$. Correlation curves and interferometric images with the spatial pixel size of 4.9 arcsec were analysed. The image synthesis and cleaning of compact sources were made with the Hanaoka algorithm. The images were synthesised every $10 \mathrm{~s}$. We used the data obtained in the circular polarisation channel, where 3-min oscillations are most evident (e.g. Abramenko \& Tsvetkov 1985; Shibasaki 2001; Gelfreikh et al. 1999). We selected 11 time intervals during 2005-2010 when only one welldeveloped and isolated sunspot, with a sufficiently strong magnetic field, was present on the solar disk: January 23-25, 2002 (NOAA 9787), May 02-05, 2005 (NOAA 0756), July 4, 2006 (NOAA 0898), August 15, 2006 (NOAA 0904) April 29, 2007 (NOAA 0953) and December 8, 2010 (NOAA 11131). Some of these events were discussed in our previous studies (Sych \& Nakariakov 2008; Sych et al. 2009; Sych et al. 2010).

Figure 1 shows a typical microwave image, used in the analysis. The image of the full solar disk was obtained with NoRH at $17 \mathrm{GHz}$ on $3 \mathrm{May}, 2005$, in the circular polarisation channel. There is only one active region, NOAA 10756 (marked by the rectangle in Fig. 1a), with a compact, highly polarised radio source of the L-polarity, with a polarisation degree of about $80 \%$ and a brightness temperature of about $127000 \mathrm{~K}$. Its spatial coordinates coincide with the large, symmetric sunspot seen in the magnetogram. The strength of the magnetic field was estimated with SOHO/MDI. At 01:35 UT on May 3, 2005, (Fig. 1b) the active region with the centre coordinates $(430,-60)$ was mainly unipolar, with prevailing S-polarity (dark areas) and a few isolated islands of the opposite polarity (bright areas). The value of the magnetic field was about $2700 \mathrm{G}$. The polarised radio source at $17 \mathrm{GHz}$ (dashed contour lines), corresponding to the extraordinary electromagnetic wave, synthesised at 02:45 UT, basically coincides with the region of the strong magnetic field.

Series of UV and EUV images on May 4, 2005, and December 8, 2010, were obtained with the Transition Region and Coronal Explorer (TRACE, Handy \& Acton 1999) and the 

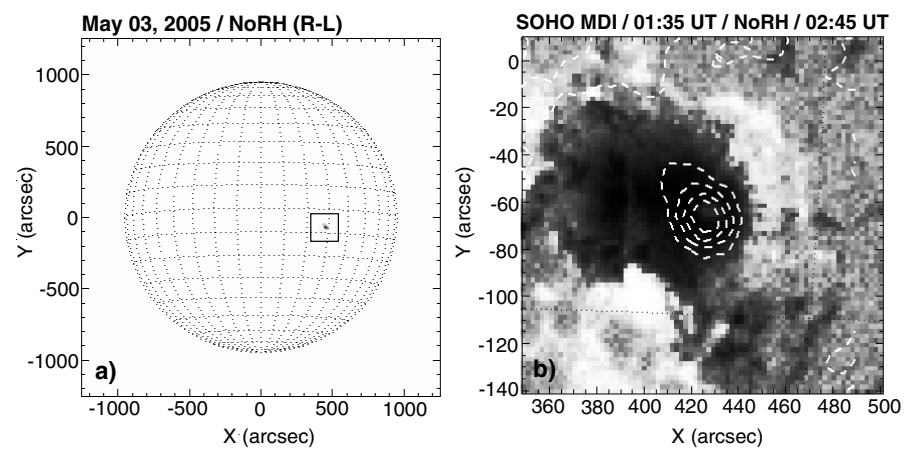

Fig. 1. Left panel: full-disk microwave polarisation image of the Sun at $17 \mathrm{GHz}$ on May 03, 2005, at 02:45 UT. The rectangle shows the radio source associated with the sunspot. Right panel: zoomed image of the radio source shown in the left panel; the dashed contours show the L-polarisation image overlapping with the SOHO/MDI magnetogram taken at 01:35 UT. The grey scale indicates the polarity of the magnetic field.

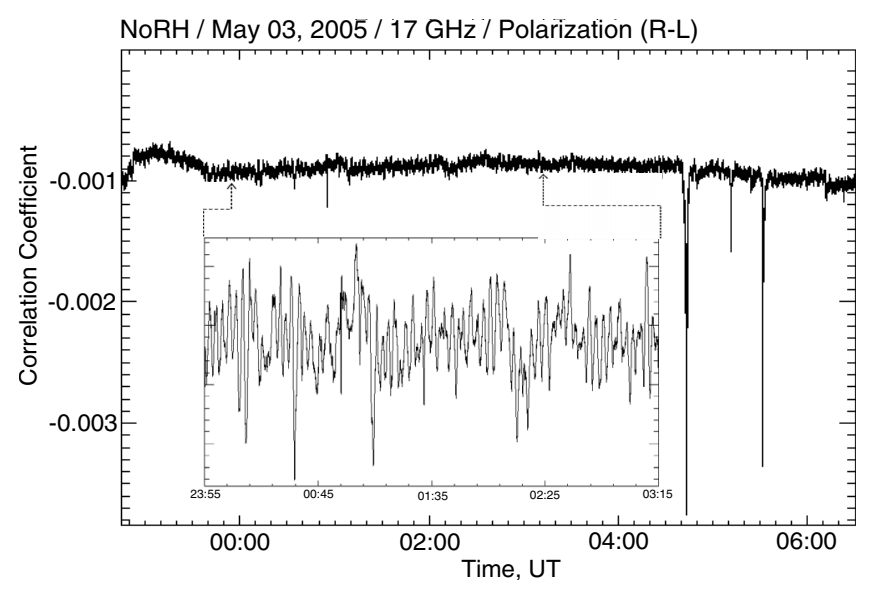

Fig. 2. Correlation curve of $\mathrm{NoRH}$ in the circular polarisation (R-L) channel. The zoomed time interval, shown in the rectangle, shows the smoothed 3-min radio oscillation profile between 23:55 and 03:15 UT.

Atmospheric Imaging Assembly (AIA) of the Solar Dynamic Observatory (SDO, Title et al. 2006), respectively. The TRACE data were processed using standard procedures: to remove the background and spike-like brightening caused by cosmic rays, to calibrate, and where it was necessary to make normalisation of the exposure time, and the removal of differential rotation during the observation period. The SDO images were obtained from level 1 data at nine wavelengths: 171, 193, 211, 304, 131, 335, 94, 1600 and $1700 \AA$, with the standard processing such as badpixel removal, despiking, and flat-fielding and centring the images in a temporary data cube.

\section{Methods}

The analysis of transient spatially-varying imaging datasets is a nontrivial task that requires dedicated tools. In our study, we applied the techniques of the wavelet transform with the Morlet mother function (Torrence \& Compo 1998) and the PWF (Sych \& Nakariakov 2008). The PWF technique allows us to make narrowband maps of power, amplitude and phase distribution of oscillation sources at selected harmonics, and obtain their temporal dynamics. The data were prepared with the web-based interactive system for the remote processing of imaging datasets (http://pwf.iszf.irk.ru, Sych et al. 2010).

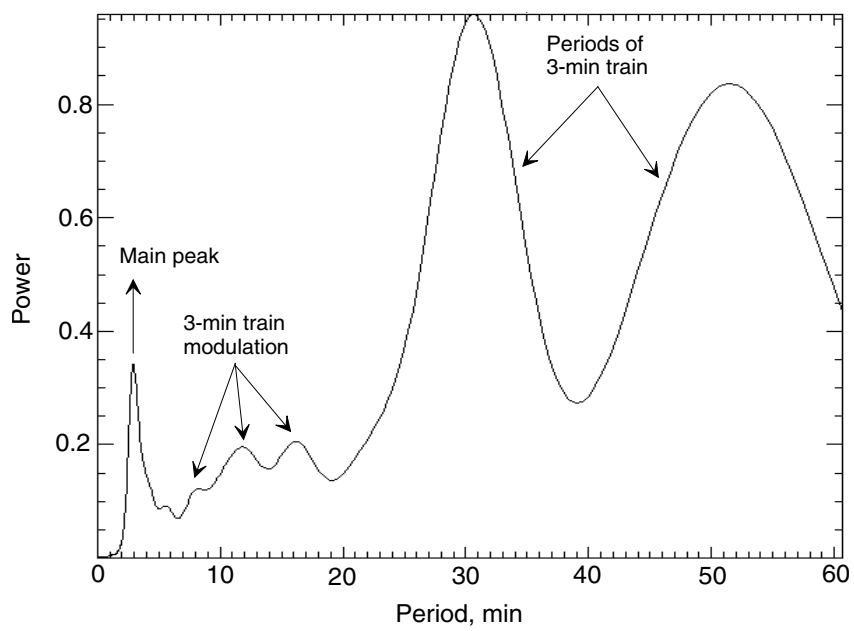

Fig. 3. Global wavelet spectrum of the NoRH correlation curve on May 3, 2005. Arrows indicate the main peaks in the spectrum.

The transient increase in the oscillation power, which leads to the local increase in the corresponding wavelet coefficients, was analysed with the method of wavelet skeletons (Mallat 1999). The method is based on extracting only those coefficients in the wavelet decomposition whose complex amplitudes are highest. Periodicities in a real signal, decomposed to a set of frequency harmonics of different amplitudes, form local peaks in the wavelet spectrum. Mapping their skeletons extracts the most-powerful harmonics and tracks their time- and frequency drifts. One can construct two types of skeletons, considering the behaviour of wavelet coefficient extrema either in the frequency or the time domains. In this study, we used the time skeletons, applying the following algorithm:

1. we constructed power wavelet spectrum with a temporal Morlet mother function;

2. we identified the frequency and time domains in the wavelet spectrum where the oscillations take place;

3. all local maxima of wavelet coefficients along the time axis in the selected time-frequency region were determined;

4. the same is repeated for global maxima;

5. finally we obtained the wavelet skeleton that combines the local and global maps.

\section{Results}

\subsection{Temporal structure of the 3-min microwave oscillations}

Figure 2 shows the profile of the NoRH correlation curve obtained in the circular polarisation channel (R-L) between 22:45, 3 May 2005, and 06:30 UT, 4 May 2005, with 1-s cadence. The relative amplitude of the oscillations is about $3-10 \%$, and reaches $17 \%$ in some peaks. The 3 -min oscillations, clearly seen in the correlation curve, are mainly related to the variation of the sunspot radio emission. The correlation curve also contains several spikes, which correspond to irregular energy releases. The radio flux from the source changed slightly compared to the full Sun flux, which could be attributed to the high level of atmospheric noise. The use of NoRH correlation curves allows one to magnify the signal associated with small-scale sources on the solar disk, and hence to carry out a preliminary analysis of the oscillations without synthesising interferometric maps. For the detailed analysis, we chose the time interval between 23:55 UT, May 2 and 03:15 UT, May 3, 2005. The zoomed 

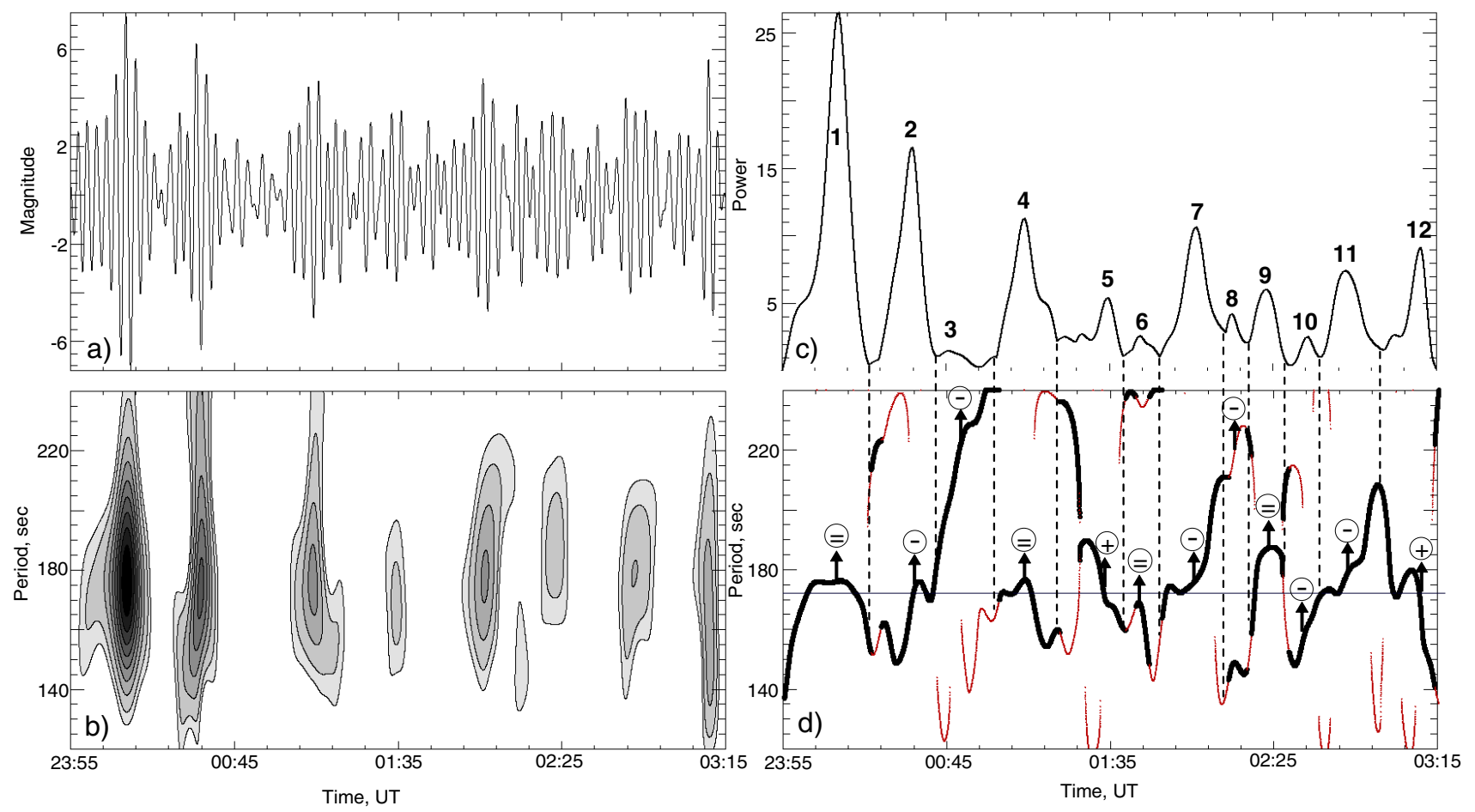

Fig. 4. Wavelet analysis of the 3-min oscillation of the microwave signal on May 3, 2005, from 23:55 to 03:15 UT. Panel a) shows the amplitudes obtained by calculating the inverse wavelet transform of the signal in the 120-240 s period range. Panel b) power distribution of the 3-min oscillation trains in the wavelet spectrum. Panel c): time evolution of the 3-min oscillation power. The numbers show the numeration of the trains. Panel d) shows the wavelet skeleton representing both global (thick lines) and local (thin lines) extrema. In the circles we show the signs of the period drifts (positive, negative or without drift). Time is shown in UT, power and amplitudes are in arbitrary units.

part of this interval, shown inside the rectangle in Fig. 2, clearly shows well-pronounced 3-min oscillations. The duration of the interval, $200 \mathrm{~min}$, and the cadence time, $1 \mathrm{~s}$, allowed us to study oscillations with periods from $3 \mathrm{~s}$ to $60 \mathrm{~min}$.

The spectral wavelet transform of the correlation curve gives us its global wavelet spectrum (GWS) shown in Fig. 3. The GWS has several well-pronounced peaks, corresponding to the periods of about $3 \mathrm{~min}$ and also to 8, 12 and $16 \mathrm{~min}$, and the long-period components of 30 and $50 \mathrm{~min}$. We point out that long-period spectral peaks have already been detected in the emission from sunspots, see, e.g. Gelfreikh et al. (1999) and Chorley et al. (2010).

We built a power wavelet spectrum of the correlation curve and extracted the periods of about 3-min, constructing the narrowband signal and wavelet skeleton shown in Fig. 4. Evidently the low-frequency amplitude modulation of the 3-min oscillation has the form of repetitive oscillation trains of varying amplitude. To obtain parameters of this modulation and visualise the power distribution of the oscillations on the period-time plane, the power wavelet spectrum was made for the narrowband signal filtered in the interval of 120 to $240 \mathrm{~s}$ periods. The wavelet spectrum is shown in Fig. 4b. Shaded contours show the power distribution normalised to the maximum value. The magnitude contours range from 0.2 to 0.9 . Darker areas represent more powerful oscillations. The irregular distribution of the power of 3-min oscillations in time and periods is clearly seen. The irregularity in the power is also seen in the main periods of the oscillations, the asymmetry of the wavelet spectral contours of the individual trains, and also in the variations of the train duration and time intervals between them. We integrated the wavelet coefficients over the frequency band, and obtained the time profile of the 3-min oscillation power (Fig. 4c). A significant periodicity in the train modulation is clearly seen. We found about twelve trains of different maximum power and duration in the analysed time interval. The train numbers are indicated at their tops.

We point out that the periods evident in GWS (Fig. 3) correspond to the characteristic times of the wave trains seen in Fig. 4c. To verify this observation, we found the time distance between the beginning and ends of each oscillation train. The duration of individual trains ranges between 7 and 21 min with an average of about $13 \mathrm{~min}$. These values coincide with the evident peaks in the GWS spectrum in the range from 8 to $16 \mathrm{~min}$. Therefore, it can be asserted that the average duration of 3-min trains is about $13 \mathrm{~min}$ with a variation close to 8,12 and $16 \mathrm{~min}$. Moreover, the long-period oscillations with periods of 30 and $50 \mathrm{~min}$ are possibly related to the characteristic time interval of the train occurrence. This possible association has also been pointed out in Chorley et al. (2010).

The observed amplitude modulation of 3 -min oscillations is accompanied by a frequency change. These changes can be visually detected from the behaviour of the power distribution in the individual trains in the frequency range (panel b, Fig. 4). To study this effect, we constructed the wavelet skeleton of the NoRH correlation curve (Fig. 4d). This allowed us to track the time and frequency dynamics of global and local extrema, and to obtain the values of frequency drifts for different phases of the sunspot activity. We can see that the period of 3-min component is not stable during the observations. There are repetitive frequency drifts with the rate from 3 to $5 \mathrm{mHz} / \mathrm{h}$, mostly occurring in the frequency band of $8.3-4.2 \mathrm{mHz}$, both increasing and decreasing the frequency. In the analysed time interval, negative drifts prevail, e.g. for trains $2,3,7,8,10$ and 11 . There are also 
Table 1. Parameters of 3-min oscillations.

\begin{tabular}{lcccccccccc}
\hline \hline No & Date & $\begin{array}{c}\text { Start } \\
\text { UT } \\
(\mathrm{h}, \mathrm{m})\end{array}$ & $\begin{array}{c}\text { End } \\
\text { UT } \\
(\mathrm{h}, \mathrm{m})\end{array}$ & $\begin{array}{c}\text { Duration } \\
\text { Observation } \\
(\mathrm{min})\end{array}$ & $\begin{array}{c}\text { Main } \\
\text { Peak } \\
(\mathrm{min})\end{array}$ & $\begin{array}{c}\text { Trains } \\
\text { Duration } \\
(\mathrm{min})\end{array}$ & $\begin{array}{c}\text { Time } \\
\text { Separation } \\
(\mathrm{min})\end{array}$ & $\begin{array}{c}\text { Negative } \\
\text { Drift } \\
(\%)\end{array}$ & $\begin{array}{c}\text { Positive } \\
\text { Drift } \\
(\%)\end{array}$ & $\begin{array}{c}\text { Variable } \\
\text { Drift } \\
(\%)\end{array}$ \\
\hline 1 & 2005-May-02 & $03: 05$ & $06: 25$ & 200 & 2.9 & 11.7 & $13,20,40,61$ & 53 & 34 & 13 \\
2 & 2005-May-03 & $23: 55$ & $03: 17$ & 200 & 2.8 & 13.3 & $8,12,16,29,52$ & 60 & 32 & 8 \\
3 & 2005-May-04 & $22: 55$ & $01: 45$ & 170 & 2.9 & 11.3 & $15,30,39,55$ & 45 & 51 & 4 \\
4 & 2005-May-05 & $00: 35$ & $03: 55$ & 200 & 2.6 & 10.2 & $13,15,21,38,51$ & 56 & 43 & 1 \\
5 & 2002-Jan.-23 & $23: 59$ & $05: 00$ & 300 & 2.8 & 10.6 & $11,18,30,37,57$ & 43 & 54 & 3 \\
6 & 2002-Jan.-24 & $01: 30$ & $04: 50$ & 200 & 3.0 & 11.1 & $11,19,27,46,67$ & 51 & 47 & 2 \\
7 & 2002-Jan.-25 & $23: 10$ & $02: 20$ & 190 & 2.8 & 11.8 & $13,23,32,46$ & 53 & 47 & 0 \\
8 & 2006-Jul.-04 & $00: 45$ & $05: 45$ & 300 & 2.8 & 11.6 & $16,33,43,58$ & 43 & 51 & 6 \\
9 & 2006-Aug.-15 & $23: 55$ & $04: 55$ & 300 & 2.8 & 13.2 & $27,36,49,74$ & 62 & 36 & 2 \\
10 & 2007-Apr--29 & $23: 15$ & $05: 35$ & 380 & 2.8 & 11.1 & $20,26,42,54$ & 48 & 46 & 9 \\
\hline
\end{tabular}

Notes. The date, the start and end time of the observation, duration of the observation (in minutes), the average period of the spectral power peak, average duration of 3-min oscillation trains, times between the trains, and the fractions of the number of trains with positive, negative and variable drifts.

intervals with positive drifts (trains 5 and 12), and with variable drifts (trains 1, 4, 6 and 9). The signs of the drifts are indicated in circles in the figure.

The times of the beginning and ends of the individual trains are shown by broken curves in Fig. 4d. Comparing the times of the power peaks of the 3-min oscillation trains (Fig. 4c) and their start and end times with the temporal dynamics of the drift skeletons, we see that the beginnings of trains and the frequency drifts coincide in the majority of cases. The period of $172 \mathrm{~s}$, corresponding to the maximum of the spectral power, determined by GWS, usually falls in the middle drift (indicated by the arrows in Fig. 4d). The drift typically begins from shorter periods of about $140 \mathrm{~s}$, and ends at about $220 \mathrm{~s}$. The formation of another train, with the frequency significantly different from the previous one, is possible before the end of the previous train. Trains 7, 8 and 9 in 02:05-02:20 UT are good examples. In individual trains, the linear, systematic frequency drift can be accompanied by some deviations, e.g. at 01:47-02:15 UT and 02:27-02:55 UT.

Similar results were found for other single sunspots analysed (02-05.05.2005 NOAA 10756, 23-25.01.2002 NOAA 9787, 04.07.2006 NOAA 10898, 15.08.2006 NOAA 2006 and 29.4.2007 NOAA 10953): 3-min oscillations are subject to persistent modulation in all analysed observations. The modulation depth varies in time by two orders of magnitude. Wavelet skeletons show both positive and negative frequency drifts. Statistical properties of the parameters of 3-min oscillations determined by the ten events are shown in Table 1.

\subsection{Spatial structure of the 3-min radio oscillations}

To study the relationship between the frequency drift and spatial changes in sunspot-associated radio sources, we considered sunspot NOAA 10756 during 3:20-05:00 UT on May 4, 2005. The GWS of the NoRH correlation curve in the $17 \mathrm{GHz}$ polarisation channel shows well-pronounced 3-min oscillations. The wavelet skeleton with the set of global and local extrema of the wavelet coefficients is shown in Fig. 5. There are three clearly visible negative frequency drifts in the analysed time interval, similar to those detected on the previous day (Fig. 4). The drift speed is about $7 \mathrm{mHz} / \mathrm{h}$. The typical duration of the drift is $40 \mathrm{~min}$. The start and end times of consecutive trains are seen to overlap. For the times indicated in Fig. 5 we constructed narrowband images of the spatial structure of the 3-min oscillation sources with the use of the PWF technique, shown in Fig. 6.

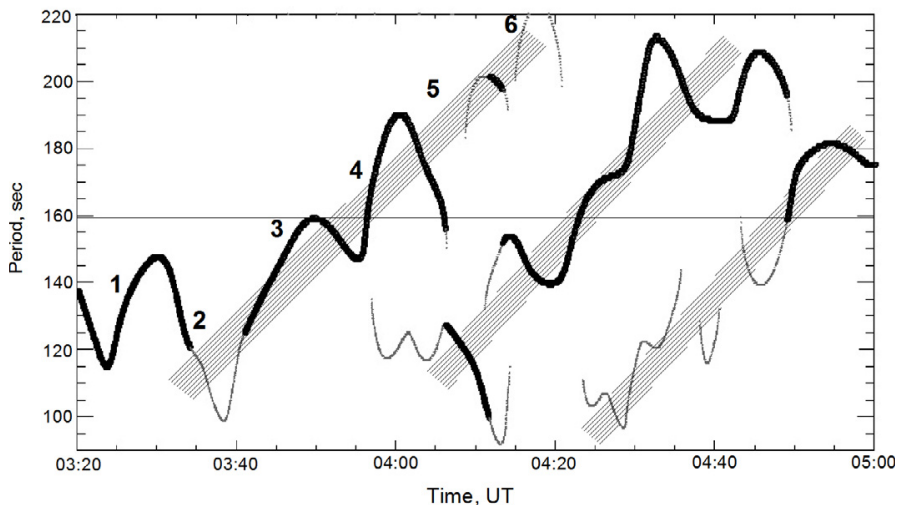

Fig. 5. Wavelet skeleton of the NoRH correlation curve in the 90-220 s period band as a function of time, obtained at $17 \mathrm{GHz}$ in the polarisation channel on May 04, 2005, 03:20-05:00 UT. The numbers indicate the times of the narrowband images shown in Fig. 6. The mesh strips indicate the prevailing trend of drifts.

Evidently there are significant spatial changes in the narrowband 3-min oscillation sources during the development of the drift: new drifts are clearly associated with the appearance of new sources of the oscillations. For example the beginning of the drift at about 3:40 UT is accompanied by the development of a new source labelled (a) in addition to the already existing source (d), which corresponds to the previous act of the frequency drift.

\subsection{Spatial structure of the 3-min oscillation trains in EUV}

To confirm the results of the frequency-spatial changes in 3-min oscillations, which were determined at the transition region in the microwave band, we analysed the data obtained at other levels of the sunspot atmosphere in the UV and EUV bands on May 4, 2005, using TRACE, and on December 8, 2010, using the SDO/AIA.

\subsubsection{The event on May 04, 2005, observed with TRACE}

Extreme ultraviolet (EUV) images of the active region NOAA 10756 with the cadence of about 9 s were obtained with TRACE at 171 $\AA$ on May 4, 2005 at 03:30-04:10 UT. After the standard calibration and pre-processing procedure, a data cube 

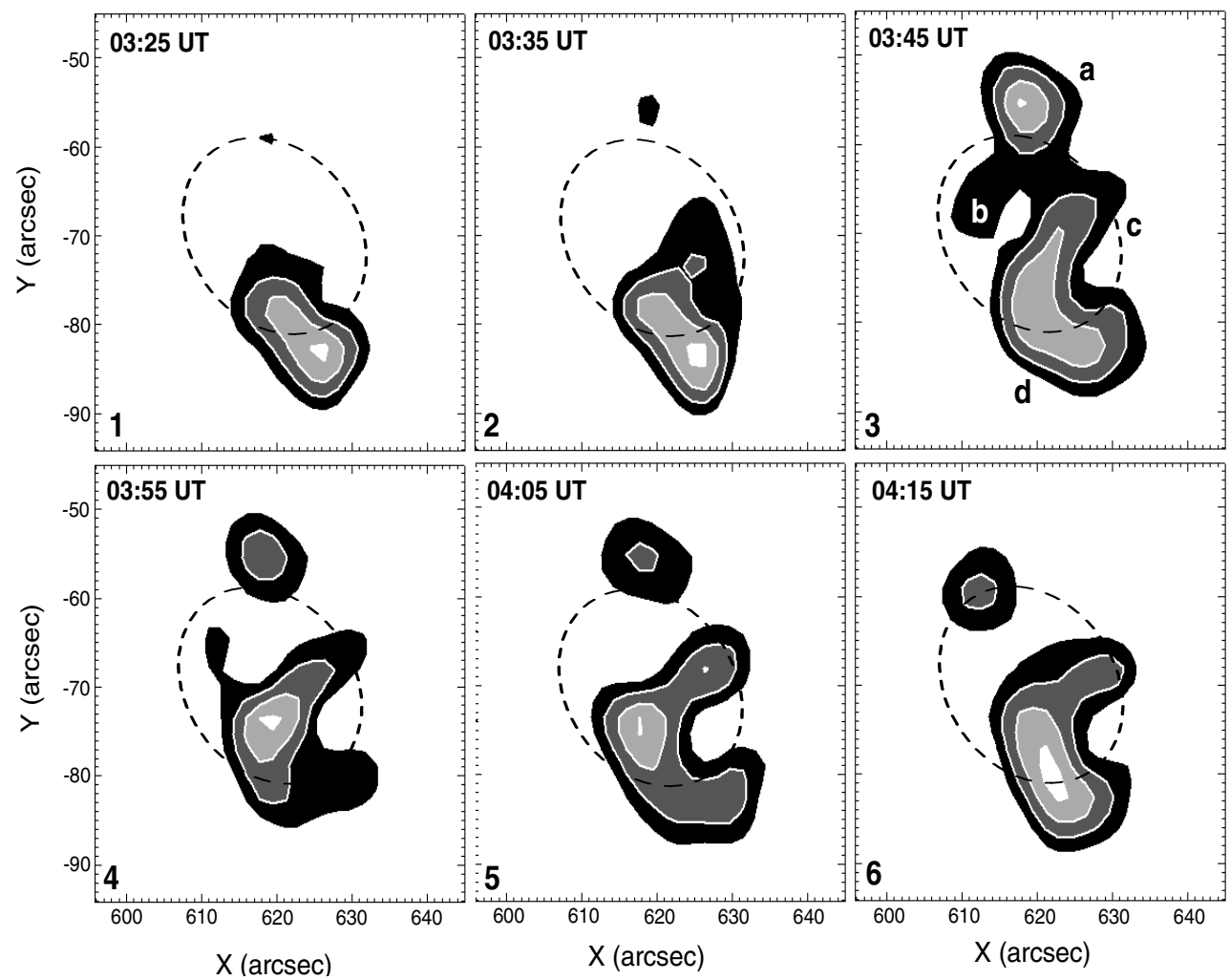

Fig. 6. Time evolution of the spatial sources of 3-min oscillations of the microwave polarisation signal from sunspot NOAA 10756, obtained with NoRH at $17 \mathrm{GHz}$ on May 04, 2005, during the 3-min frequency drift 03:20-04:30 UT. The grey colour scale shows the power of the oscillation. The dashed curve indicates the umbra-penumbra boundary. The integer numbers label the instants of time shown in Fig. 5. The letters label individual sources of the oscillations.

of the $50 \times 50$ arcsec images of the active region was obtained. A movie of the data cube clearly shows periodic EUV disturbances propagating along the plasma fan from the sunspot outwards. Integrating the data cube over the spatial coordinates we obtained the time profile of the EUV flux from the active region. The wavelet skeleton constructed in the 90-220 s period band shows a negative frequency drift of the 3-min oscillations (Fig. 7, left panel), similar to the drift detected in the microwave in the transition region (Fig. 5). However, the drift speed is about $13 \mathrm{mHz} / \mathrm{h}$, which is almost two times higher than detected at $17 \mathrm{GHz}$.

We used the PWF-method to investigate the spatial structure of the 3-min oscillation sources at different heights and their relationship to the frequency drift at about 03:40 UT, when significant changes were detected in the microwave band (Fig. 7, right panel). Evidently the power of 3-min waves concentrates in some open plasma structures (which were called "wave traces" in Sych et al. 2009, 2010). Although there is almost an order of magnitude difference in the spatial resolution of NoRH and TRACE, the 3-min oscillation sources determined in EUV have similar spatial location and shape as the sources determined in microwaves (see the details labelled by the letters a, b, c and d in Figs. 6 and 7, right panel). Like in the microwave data, the appearance of new sources of 3-min oscillations coincides with the beginning of a 30-min train and with the frequency drift.

\subsubsection{The event on December 08, 2010, observed with SDO/AIA}

The active region NOAA 11131 was observed by SDO/AIA on December 8, 2010. Sequences of $72 \times 72$ arcsec images were obtained with the 24-s cadence over the time interval 02:3003:30 UT at 1600, 1700, 171, 304, 193, 211, 131, 335 and $94 \AA$ wavelengths. This set of observational wavelengths allows us to study properties of 3-min oscillations, in particular the frequency drifts, from the photospheric up to coronal heights (Fig. 8, top panel). Applying PWF, we made data cubes of the spatial distribution of narrowband 3-min oscillations. Snapshots of the spatial structure of the oscillations are shown in the bottom panels of Fig. 8.

We see that the 3-min oscillation sources are mainly concentrated in the umbra. For lower layers of solar atmosphere, i.e. at $1700 \AA$, the sources have the shape of symmetric patches that fill the central part of the umbra almost uniformly. In the chromospheric level, i.e. at $304 \AA$, we see the fragmentation of the oscillation source that gains a spiral shape, filling up the whole umbra. In the coronal level ( $171 \AA$ ), the oscillation source is even more deformed: a part of the source has a radial sector structure, while the other part is stretched along coronal plasma loops that form the active region fan. A similar shape of the oscillation source is seen in higher-temperature emission lines, but the power of 3-min oscillations in this emission is significantly lower.

Information about frequency drifts and wave propagation can be obtained from time-distance plots, constructed for the time variation of the emission intensity along a certain onedimensional spatial slit. We show such a plot for a horizontal slit that passes through the centre of the sunspot in the $304 \AA$ data cube in Fig. 9. The wave structure of the 3-min oscillations, which propagate symmetrically from the sunspot centre and the subsequent decrease in the propagation speed and damping at the umbra-penumbra boundary are clearly visible. An analysis 
TRACE 171A, May 04, 2005

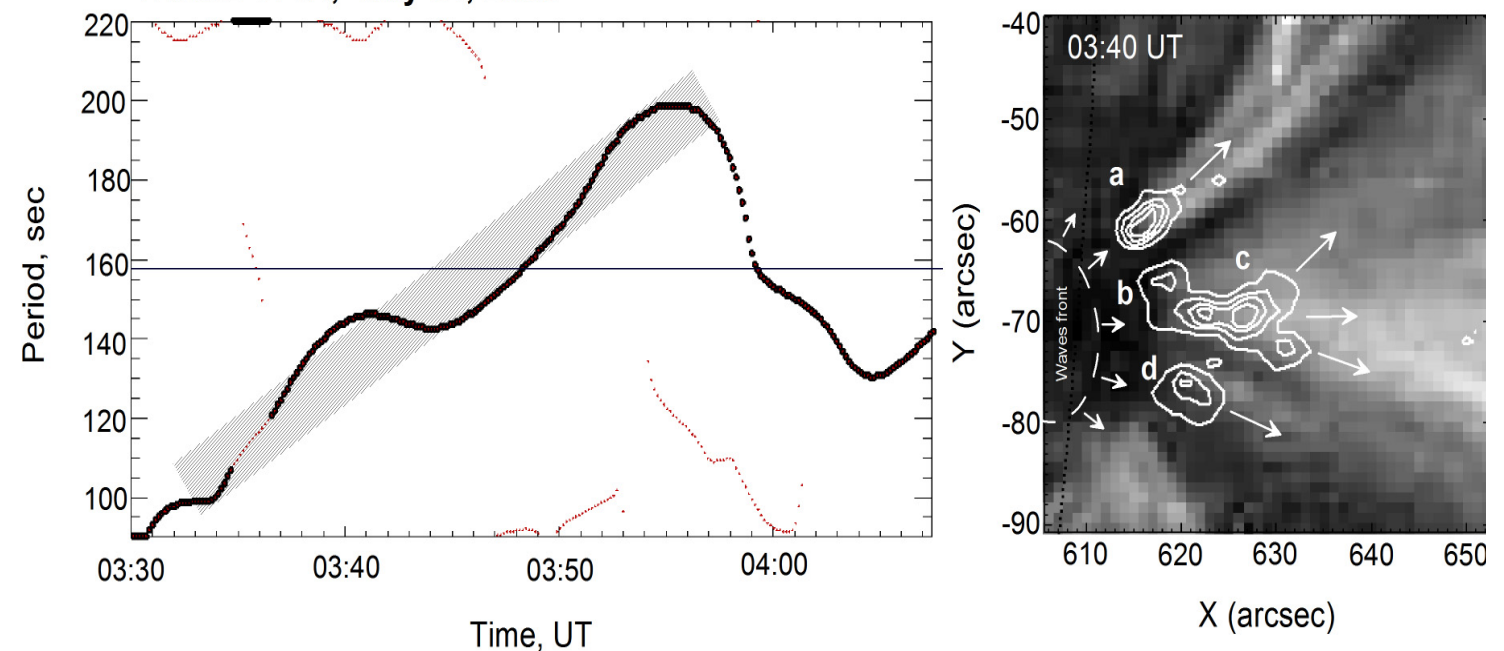

Fig. 7. Left panel: wavelet skeleton of 3-min oscillations observed in the EUV emission over sunspot NOAA 10756 on May 04, 2005, at 03:3004:08 UT. The negative trend of the frequency drift is shown by the mesh strip. Thick lines correspond to the global maxima of wavelet coefficients, and the thin lines to the local ones. Right panel: overlap of the narrowband image of 3-min EUV oscillation sources (shown by white contours) on the integral EUV image at 03:40 UT. The letters a, b, c and d correspond to the fine 3-min oscillation radio sources in Fig. 6 at 03:45 UT. The arrows indicate the apparent direction of the wave propagation.

of the time characteristics of the signals was performed separately for each observational wavelength in the same fashion as for the TRACE data (Sect. 4.3.1).

Figure 10 shows the amplitude and phase modulation of 3-min oscillations for three wavelengths, 1700, 304 and $171 \AA$, which correspond to three different heights, and their wavelet skeletons. As in our previous findings, the oscillation trains last typically from 8 to $20 \mathrm{~min}$ (with the average of about $12 \mathrm{~min}$ ), showing partial overlap. Times between individual trains are about $30 \mathrm{~min}$. Wavelet skeletons show that the rising phase of the trains is accompanied with negative frequency trains. The average period of 3 -min oscillations is $155 \mathrm{~s}$ at $1700 \AA$, $153 \mathrm{~s}$ at $304 \AA$ and 162 s at $171 \AA$. Negative frequency drifts dominate in all observational channels. The drift speed at the photospheric level $(1600 \AA, 1700 \AA)$ is about $4-5 \mathrm{mHz} / \mathrm{h}$, at the chromospheric level (304 $\AA$ ) about $5-7 \mathrm{mHz} / \mathrm{h}$, and at microwaves (NoRH, $17 \mathrm{GHz}) 5.6-8 \mathrm{mHz} / \mathrm{h}$ and in the corona $(171,193$, 211 and $131 \AA$ ) about $11-13 \mathrm{mHz} / \mathrm{h}$. Accordingly, the absolute value of the drift speed increases by almost two orders of magnitude during the propagating from the sub-photospheric levels to the corona. In higher temperature emission (335 and $94 \AA$ ) the 3-min signal is weak, and hence it is impossible to obtain reliable information about the drifts.

\section{Discussion}

Using high-quality EUV and microwave data obtained with SDO/AIA, TRACE and NoRH and modern analytical techniques (PWF-method and wavelet skeletons), we studied complex temporal dynamics of 3-min oscillations above sunspots. The dynamics include variations in height, space, time, and frequency. Spatially-integrated signals obtained at different heights and with different instruments show a common behaviour: the oscillations experience amplitude and frequency modulation, which is accompanied by the change of the spatial sources. The amplitude modulation has the form of wave trains, with a duration of 7 and $21 \mathrm{~min}$ and a repetition period of about 30-60 min. The frequency modulation has the form of frequency (or period) drifts in the 2-4 min period range. Typically, the beginning and end times of the drifts coincide with the trains of the oscillations, which indicates their phenomenological relationship. Mainly negative drifts (the gradual increase in the period of 3-min oscillations) are observed. Sometimes two drifts co-exist, i.e. during the end of the previous drift, when the frequency is in the low-frequency part of the 3-min spectrum, near $200 \mathrm{~s}$, a new drift appears near $160 \mathrm{~s}$. The development of new 3-min oscillation trains is typically accompanied by the appearance of new spatial sources (the regions of the enhanced power of 3-min oscillations in the horizontal plane) in the sunspot atmosphere. In the corona, spatial sources of 3-min oscillations develop along certain magnetic flux tubes of the magnetic fans above the sunspot. Narrowband time-distance plots show that in those structures 3-min oscillations have a form of outward propagating waves. This behaviour agrees well with recent numerical modelling (Botha et al. 2011) and the analysis of imaging and spectral EUV data (e.g. De Moortel 2009; Verwichte et al. 2010). Moreover, there are coronal sources of another type: compact, of a symmetric, patch-like shape, which can perhaps be associated with the magnetic flux tubes with a narrow angle to the line-ofsight.

\subsection{A possible mechanism for frequency drifts}

One of the possible mechanisms explaining the observed properties of 3 -min oscillations oscillation is the response of the sharply stratified atmosphere to pulses coming from lower regions. The pulses can be excited by magnetic reconnection, granulation or other explosive or impulsive events that take place in sub-photospheric, photospheric or lower chromospheric regions.

Consider a gravitationally stratified plane atmosphere with a vertical magnetic field. We assume that $p_{0}(z)$ and $\rho_{0}(z)$ are the plasma pressure and density, respectively; and the temperature, $T_{0}$, is constant. Then sound speed, $c_{\mathrm{s}}=\sqrt{\gamma p_{0} / \rho_{0}}$, is also constant. The pressure scale height is

$H=\frac{k_{B} T_{0}}{\hat{m} g}$, 

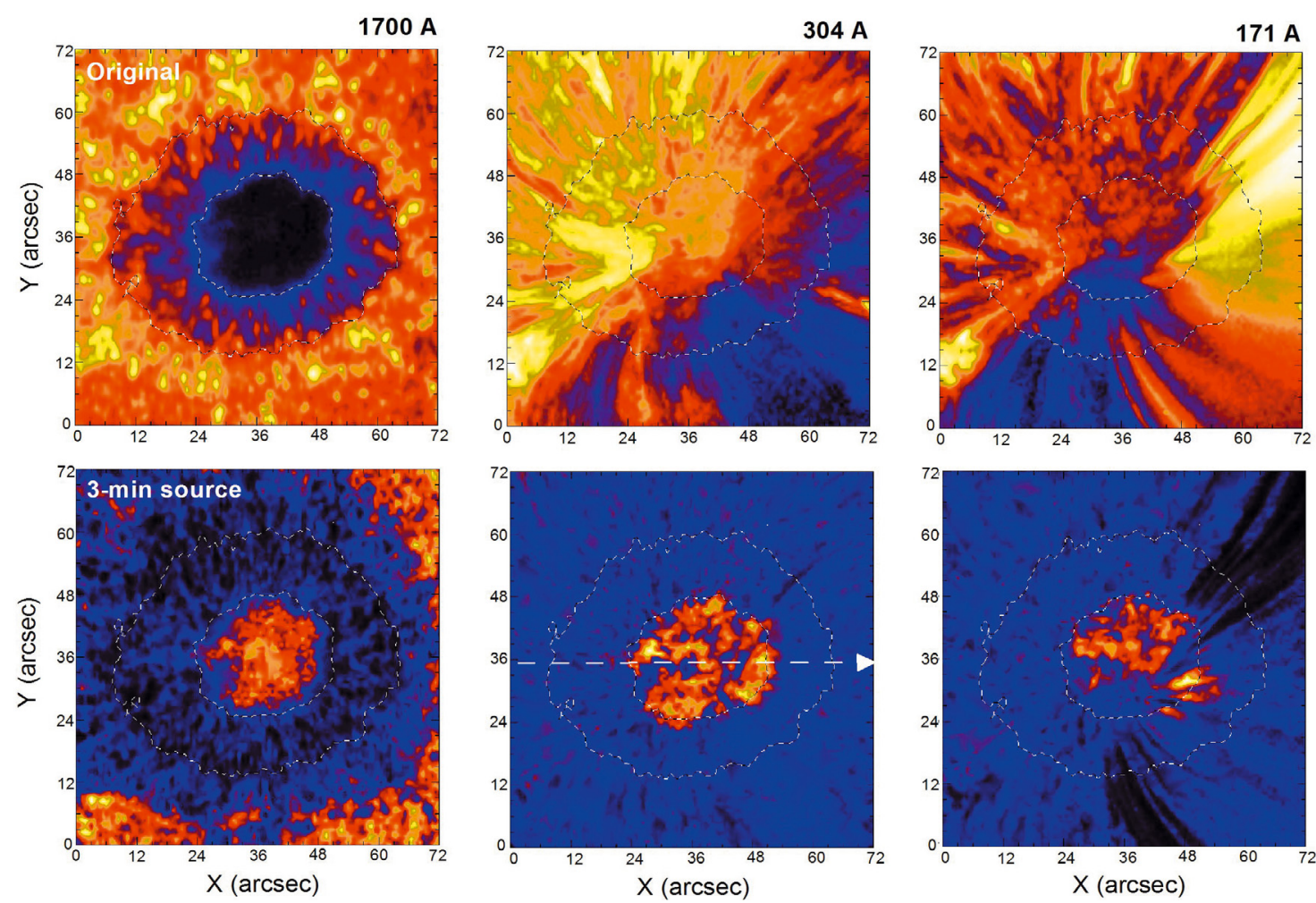

Fig. 8. Top panels: images of NOAA 11131 at $1700 \AA$ (photosphere), $304 \AA$ (chromosphere) and $171 \AA$ (corona) on December 8, 2010, at 02:30 UT. Bottom panels: spatial distribution of 3-min oscillations, obtained by filtering in the $5.2-9.5 \mathrm{mHz}$ band. Thin dotted lines indicate the umbra-penumbra boundary at the photospheric level. The thick dashed line in the narrowband image at $304 \AA$ shows the slit of the time-distance plot.

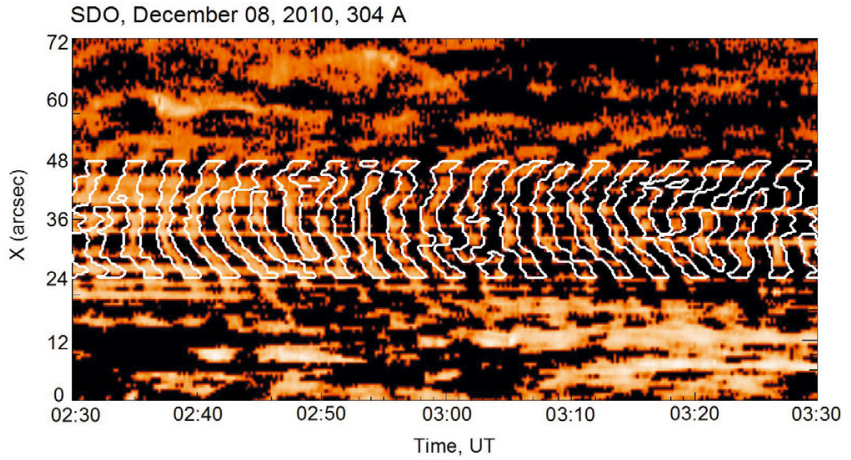

Fig. 9. Time-distance plot of the active region NOAA 11131 on December 8, 2010, obtained by SDO/AIA at 304 A. The intensity oscillations are presented in the logarithmic scale. The white contours show the narrowband wave fronts in the $5.2-9.3 \mathrm{mHz}$ band. The spatial slit is positioned across the sunspot, through its centre, and is shown in Fig. 8.

where $k_{B}$ is the Boltzmann constant, $g$ is the gravitational acceleration and $\hat{m}$ is the mean molecular weight. This model of the solar lower atmosphere is obviously oversimplified, but we use it for illustration purposes only.

The propagation of linear sound waves in the stratified medium is governed by the Klein-Gordon equation, which implies the acoustic cut-off frequency

$\omega_{\mathrm{c}}=\frac{c_{\mathrm{s}}}{2 H}$.
Waves with frequencies higher than $\omega_{\mathrm{c}}$ can propagate upwards, while the lower-frequency waves are evanescent. However, the evolution of an initially broadband pulse leads to a formation of an oscillatory wake (Lamb 1908; Rae \& Roberts 1982):

$u_{1}=u_{0} J_{0}\left(\sqrt{\omega_{\mathrm{c}}^{2} t^{2}-\frac{z^{2}}{4 H^{2}}}\right) \mathcal{H}\left(\omega_{\mathrm{c}} t-\frac{z}{2 H}\right) \exp \frac{z}{2 H}$,

where $u_{1}$ is the vertical component of the velocity, $u_{0}$ is the initial amplitude, $J_{0}$ is the Bessel function of the zeroth order, and $\mathcal{H}$ is the Heaviside function. The time is measured from the time when the pulse was launched.

Because the chromospheric cut-off period is about 3-min the observed oscillations can be explained as wakes behind the pulses that propagate along the vertical magnetic field (Fleck \& Schmitz 1991; Kalkofen et al. 1994; Sutmann \& Ulmschneider 1995; Kuridze et al. 2009). However, each active region may have a fine structure in the horizontal direction in the form of flux tubes with different plasma temperatures, because the strong magnetic field prohibits thermal conduction across the field. Then, the pulses propagating in different parts of the active region may set up the wakes with different oscillating periods. The radio observations may not resolve the fine horizontal structure of the oscillations because of the insufficient spatial resolution. Then the oscillations with different frequencies will be superimposed, and the resulting observed oscillation may have a complex structure.

In order to illustrate this scenario, we plot in Fig. 11 the linear superposition of two wakes, which could be formed by two 
R. Sych et al.: Frequency drifts of 3-min oscillations in radio and EUV above sunspots

Solar Dynamic Observatory (SDO), December 08, 2010
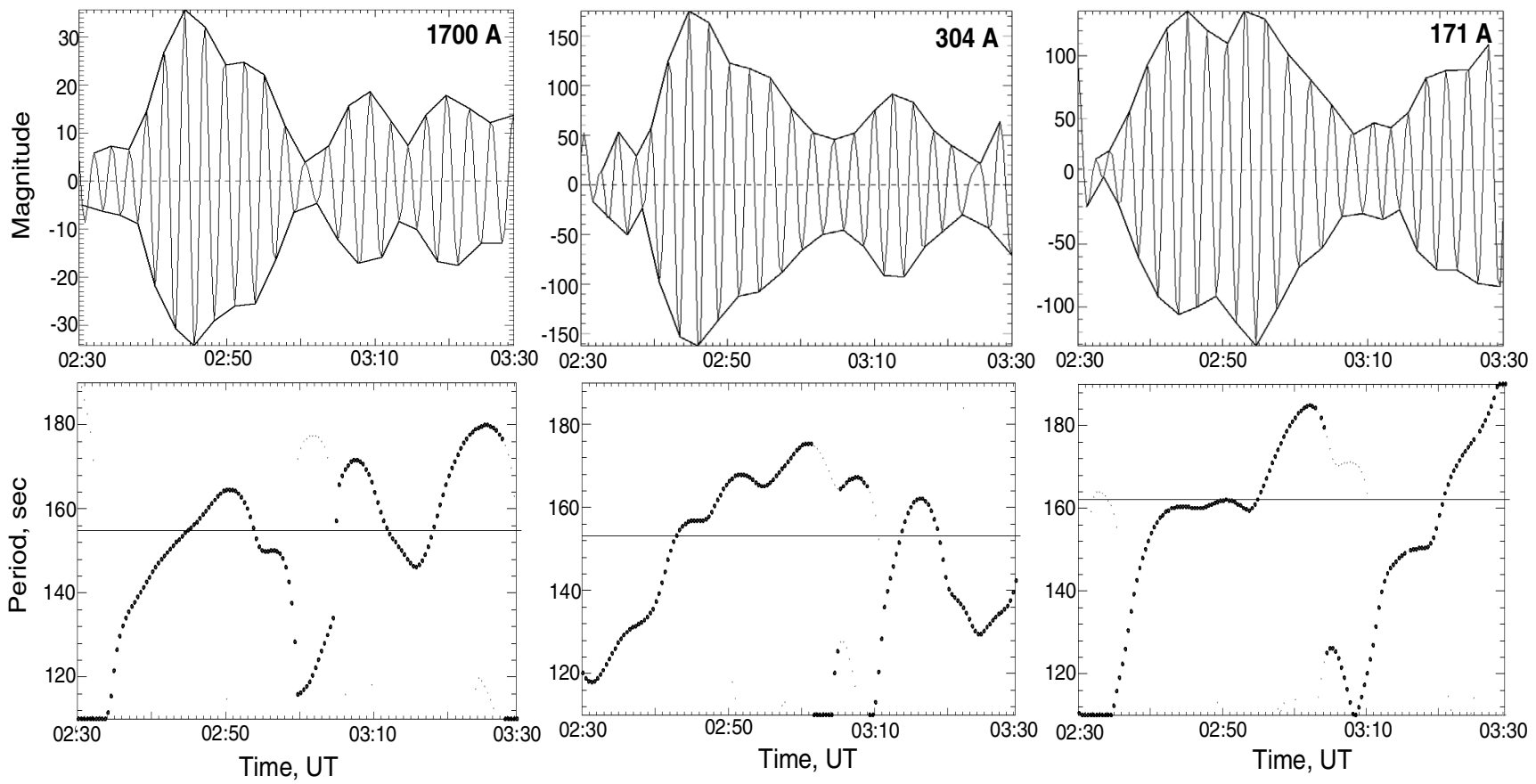

Fig. 10. Top panels: time profiles of 3-min oscillation trains in the emission intensity of the 1700, 304 and 171 A signals obtained with SDO/AIA on December 8, 2010, at 2:30-03:30 UT. Envelopes of the trains are shown by the thick curves. Bottom panels: wavelet skeletons of the signals. The continuous line shows the period corresponding to the maximum spectral power.
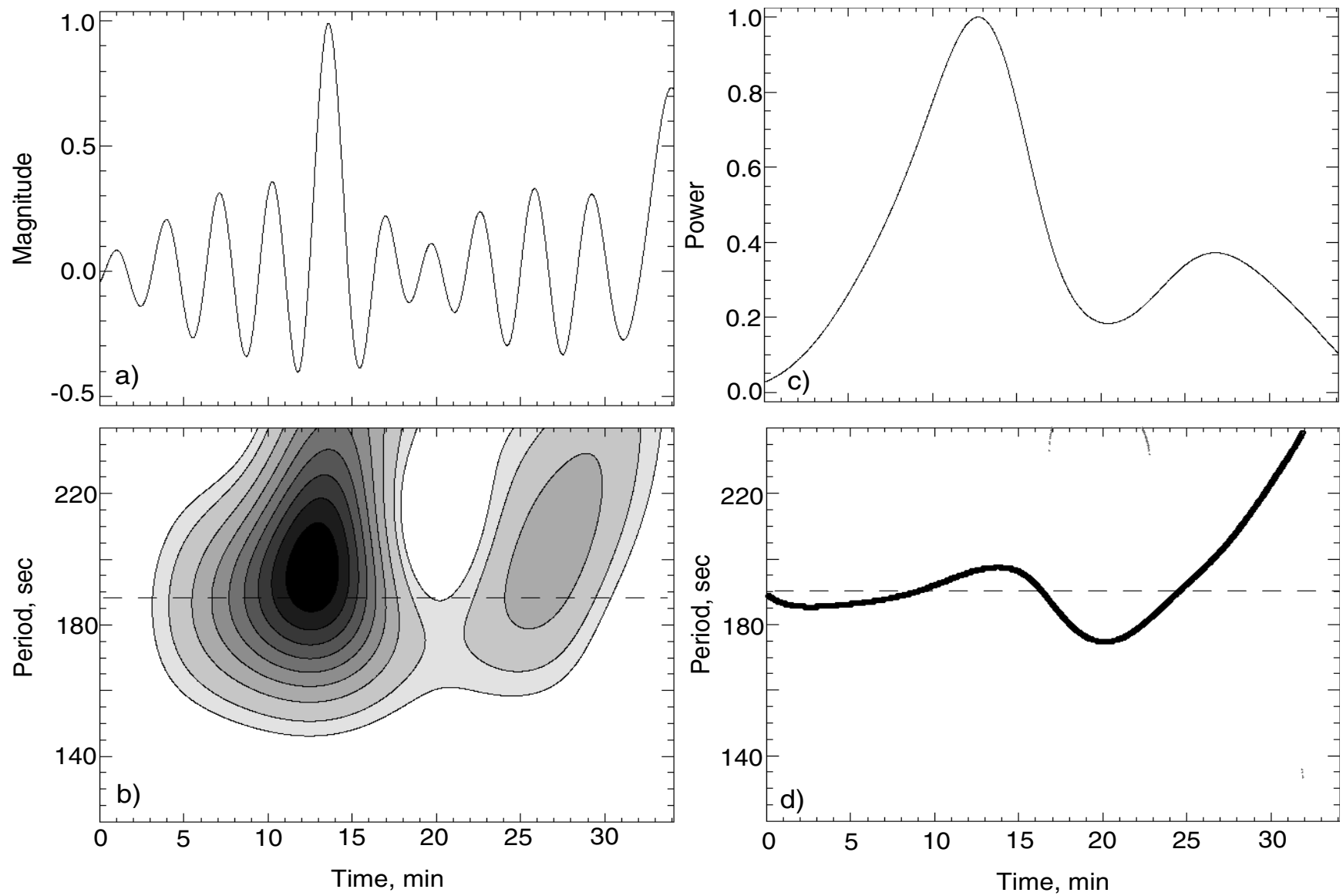

Fig. 11. Model of the observed behaviour of 3-min oscillations: superposition of two oscillatory wakes formed in two different magnetic flux tubes in a sunspot's umbra. Panel a): the signal; panel b): its wavelet spectrum; panel c): time dependence of the oscillation power; panel d): wavelet skeleton of the signal. 
acoustic pulses propagating along two different flux tubes in the umbra. The ratio of the cut-off frequencies in these two regions is taken to be 0.8 . The second pulse was launched $810 \mathrm{~s}$ later than the first pulse. We also show the wavelet spectrum of the signal, time variation of the oscillation power, and the wavelet skeleton with the global maximum. The time interval between the wave trains is about $14 \mathrm{~min}$, which actually is the interval between the consecutive pulses. The oscillation period slightly increases (decreases) before (after) the maximum of the oscillation amplitude. Thus, the behaviour of the modelled oscillation is very similar to the observed behaviour of 3-min oscillation trains.

\section{Conclusions}

We analysed 3-min oscillations in the NoRH polarisation correlation curves at $17 \mathrm{GHz}$, obtained for eleven time intervals when there was only one powerful sunspot on the solar disk. The relative amplitude of 3-min oscillations was about 3-8\% and sometime reached $17 \%$. The spectral analysis showed significant peaks in the range of $2-4$ min with a power concentration near $3 \mathrm{~min}$ period. The 3 -min oscillations experience amplitude modulation in the form of periodic wave trains, lasting for 8-20 min with $13 \mathrm{~min}$ in average. The repetition rate of the trains is $30-50 \mathrm{~min}$. There were also low-frequency peaks in the spectrum, corresponding to the periods of 10-20 min, and 30-60 min. Apparently these long-period oscillations coincide with the characteristic times of the 3-min oscillation trains.

In addition, 3-min oscillations are subject to frequency modulation: their period varies in the range 90-240 s. During the oscillation train, the frequency modulation often has the form of a steady frequency drift. The beginning and end of the drift coincide with the beginning and end of the amplitude modulation. In the majority of cases, the negative drift, i.e. when the 3 -min oscillation period gradually increases, is observed. In different trains of 3-min oscillations observed in the same sunspot, there can be both positive and negative drifts. The start and end times of consecutive drifts can overlap. This behaviour is seen at all levels of the sunspot atmosphere. The speed of the drift is 4-5 mHz/h in the photosphere, $5-8 \mathrm{mHz} / \mathrm{h}$ in the chromosphere, and $11-13 \mathrm{mHz} / \mathrm{h}$ in the corona. Therefore the drift speed apparently grows with height. In addition to the gradual drift, the instant frequency of 3-min oscillations shows positive and negative fluctuations.

An analysis of the spatial structure of 3-min sunspot oscillations observed in microwaves and EUV shows that the start of drifts coincides with the appearance of the oscillation sources (the regions with the enhanced power of 3-min oscillations).

The observed spatial-frequency-temporal properties of 3-min oscillations can be interpreted in terms of two simultaneously-operating mechanisms. The first effect is connected to the negative frequency drift caused by the dispersive evolution of upward propagating sub-photospheric pulses, guided by the sunspot magnetic field. The repetition rate of the pulses (30-60 min) is similar to the long-period oscillations of sunspots, which suggests their possible association. The second effect is the spatial separation of the propagating pulse by several individual magnetic flux tubes of different curvature, temperature and line-of-sight angles. In particular, the frequency drifts may take place because of the changes in the tube curvature with height.

Acknowledgements. The work was supported by the grants RFBR 10-02-00153 and 11-02-10000-k and Lavrentiev grant SB RAS (2010-2011). R.S. is supported by the Chinese Academy of Sciences Visiting Professorship for Senior
International Scientists No. 2010T2J24. T.Z. is supported by the Austrian Fonds zur Förderung der wissenschaftlichen Forschung (project P21197-N16) and the Georgian National Science Foundation (grant GNSF/ST09/4-310). The TRACE, SDO and NoRH data were obtained from the TRACE, SDO and NoRH databases. The authors acknowledge the TRACE, SDO and NoRH consortia for operating the instruments and performing the basic data reduction, and especially for the open data policy. Wavelet software was provided by C. Torrence and G. Compo, and is available at URL: http://paos. colorado. edu/research/wavelets/.

\section{References}

Abramenko, V. I., \& Tsvetkov, L. I. 1985, Bull. Crim. Astrophys. Obs., 73, 53 Balthasar, H., Martinez Pillet, V., \& Schleicher, H. 1998, Sol. Phys., 182, 65

Bard, S., \& Carlsson, M. 1997, SOHO Workshop: The Corona and Solar Wind Near Minimum Activity, ESA SP, 404, 189

Bellot Rubio, L. R., Collados, M., Ruiz Cobo, B., \& Rodriguez Hidalgo, I. 2000, ApJ, 534, 989

Bogdan, T. J. 2000, Sol. Phys., 192, 373

Botha, G. J. J., Arber, T. D., Nakariakov, V. M., \& Zhugzhda, Y. D. 2011, ApJ, 728,84

Brković, A., Ruedi, I., Solanki, S. K., et al. 2000, A\&A, 353, 1083

Chorley, N., Hnat, B., Nakariakov, V. M., Inglis, A. R., \& Bakunina, I. A. 2010, A\&A, 513, A27

De Moortel, I. 2009, Space Sci. Rev., 149, 65

Elliott, I. 1969, Sol. Phys., 6, 28

Fleck, B., \& Schmitz, F. 1991, A\&A, 250, 235

Fludra, A. 2001, A\&A, 368, 639

Gelfreikh G. B., Grechnev, V., Kosugi, T., et al. 1999, Sol. Phys., 185, 177

Gelfreikh, G. B., Shibasaki, K., Nagovitsyna, E. Yu., et al. 2004, IAU Symp. 223 (Cambridge University Press), 245

Gurman, J. B., Leibacher, J. W., Shine, R. A., et al. 1982, ApJ., 253, 939

Handy, B. N., \& Acton, L. W. 1999, Sol. Phys., 187, 229

Harrison, R. A., Lang, J., Brooks, D. H., et al. 1999, A\&A, 351, 1115

Kalkofen, W., Rossi, P., Bodo, G., et al. 1994, A\&A, 284, 976

Kobanov, N. I., Kolobov, D. Y., \& Chupin, S. A. 2008, Astron. Lett., 34, 133

Kobanov, N. I., Kolobov, D. Y., Chupin, S. A., \& Nakariakov, V. M. 2011, A\&A, 525, A41

Kuridze, D., Zaqarashvili, T. V., Shergelashvili, B. M., et al. 2009, A\&A, 505, 763

Lamb, H. 1908, Proc. Lond. Math. Soc., Ser., 2, 7, 122

Lites, B. W. 1986, ApJ, 301, 1005

Lites, B. W. 1992, in Sunspots: Theory and Observations, ed. J. H. Thomas, \& N. O. Weiss (NATO ASJ Ser. C, 375; Dordrecht: Kluwer), 261

Leibacher, J. W., \& Stein, R. F. 1981, In The Sun as a Star, ed. S. Jordan, NASA SP, 450, 263

Leibacher, J., Gouttebroze, P., \& Stein, R. F. 1982, ApJ, 258, 393

Liu, S. Y. 1974, ApJ, 189, 359

Mallat, S. 1999, A Wavelet Tour of Signal Processing, 2nd ed. (San Diego: Academic Press)

Murawski, K., \& Zaqarashvili, T. V. 2010, A\&A, 519, A8

Muzy, J. F., Bacry, E., \& Arneodo, A. 1993, Phys. Rev. E, 47, 875

Nakajima, H., Nishio, M., Enome, S., et al. 1994, IEEE Proceedings, 82, 705

Nakariakov, V. M., Inglis, A. R., Zimovets, I. V., et al. 2010, Plasma Physics and Controlled Fusion, 52, 12

Nindos, A., Alissandrakis, C. E., Gelfreikh, G. B., et al. 2002, A\&A, 386, 658

Norton, A. A., Ulrich, R. K., Bush, R. I., et al. 1999, ApJ, 518, L123

Orrall, F. Q. 1966, ApJ, 143, 917

Percival, D. B., \& Walden A. T. 2000, Wavelet methods for time series analysis (Cambridge University Press)

Rae I. C., \& Roberts, B. 1982, ApJ, 256, 761

Rendtel, J., Staude, J., \& Curdt, W. 2003, A\&A, 410, 315

Rioul, O., \& Vetterli, M. 1991, Wavelet and Signal Processing, IEEE Signal Processing Magazine

Shibasaki, K. 2001, ApJ, 550, 1113

Sutmann, G., \& Ulmschneider, P. 1995, A\&A, 294, 232

Sych, R. A., \& Nakariakov, V. M. 2008, Sol. Phys., 248, 395

Sych, R., Nakariakov, V. M., Karlicky, M., et al. 2009, A\&A, 505, 791

Sych, R. A., Nakariakov, V. M., Anfinogentov, S. A., \& Ofman, L. 2010, Sol. Phys., 266, 349

Title, A. M., Hoeksema, J. T., \& Schrijver, C. J. 2006, 36th COSPAR Scientific Assembly, CD ROM 2600

Torrence, C., \& Compo, G. P. 1998, Bull. Am. Meteorol. Soc., 79, 61

Tziotziou, K., Tsiropoula, G., \& Mein, P. 2002, A\&A, 381, 279

Verwichte, E., Marsh, M., Foullon, C., et al. 2010, ApJ, 724, L194

Zaqarashvili, T. V., Murawski, K., Khodachenko, M. L., \& Lee, D. 2011, A\&A, 529, A85

Zhugzhda, Y. D., Balthasar, H., \& Staude, J. 2000, A\&A, 355, 347 